\title{
Acute Dacryocystitis with Empyema of the Lacrimal Sac: Is Immediate Endoscopic Dacryocystorhinostomy Justified?
}

Otolaryngology-

Head and Neck Surgery

2014, Vol. 150(6) 107I-1077

(C) American Academy of

Otolaryngology-Head and Neck

Surgery Foundation 2014

Reprints and permission:

sagepub.com/journalsPermissions.nav DOI: $10.1177 / 0194599814527236$ http://otojournal.org

(S)AGE

\author{
Davide Lombardi, MD', Davide Mattavelli, MD', \\ Remo Accorona, MD', Raffaele Turano, MD², \\ Francesco Semeraro, MD², Anna Bozzola, MD', \\ and Piero Nicolai, MD'
}

No sponsorships or competing interests have been disclosed for this article.

\begin{abstract}
Objectives. To evaluate the efficacy of endoscopic dacryocystorhinostomy (Endo-DCR) in the treatment of acute dacryocystitis with lacrimal sac empyema (ADLSE).
\end{abstract}

Design. Case series with chart review.

Setting. Academic tertiary center.

Patients. The study included 26 consecutive patients who underwent Endo-DCR for ADLSE between August 2005 and December 2013.

Main Outcome Measures. The success of the procedure was defined as complete complaint relief and DCR patency. Data on the time from referral to surgery, postoperative complications, and revision surgery are also reported.

Results. The present patient series included 4 males (I5.4\%) and 22 females (84.6\%) (mean age, 66 years). The mean time between referral and surgery was 0.88 days and the mean follow-up time was 29 months. All patients showed immediate relief from symptoms, with no ADLSE recurrences. Complete success was achieved in 25 (96.2\%) cases; the only failure was in a patient who had previously undergone radioiodine treatment. In this case, revision Endo-DCR was not successful. The only perioperative complication (3.8\%) was epistaxis in a patient who required revision surgery under general anesthesia. The definitive success rate was $96.2 \%$ after primary and revision surgery.

Conclusions. Endo-DCR enables rapid resolution of ADLSE with a very high success rate. Immediate surgery may reduce the risk of skin fistulization and/or orbital complications. DCR shrinkage and lacrimal obstruction are unlikely with Endo-DCR since the procedure is performed on an enlarged sac. The main advantage of Endo-DCR, compared with external DCR, is the absence of a skin incision in an inflamed and infected field.

\section{Keywords}

acute dacryocystitis, endoscopic dacryocystorhinostomy, lacrimal sac empyema

Received August 20, 20I3; revised February 4, 20I4; accepted February 18, 2014.

\section{Introduction}

Epiphora, eyelid and lacrimal sac swelling, purulent secretion from canaliculi, and recurrent dacryocystitis are typical complaints in patients affected by occlusion of the lacrimal pathway. Acute dacryocystitis is a presenting modality in a substantial proportion of patients with lacrimal pathway obstruction and is characterized by a well-defined swelling at the medial canthus. When this swelling has ill-defined borders and the sac is massively dilated and covered by hyperemic and congested skin that is possibly associated with eyelid edema (Figure I), the term acute dacryocystitis with lacrimal sac empyema ( $A D L S E$ ) would seem to be the more appropriate description of this condition.

Historically, conventional treatment for ADLSE consisted of prolonged systemic antibiotics together with warm compresses and percutaneous incision and drainage of the lacrimal sac empyema. ${ }^{1}$ Surgical treatment, that is, external dacryocystorhinostomy (Ex-DCR), ${ }^{2}$ was generally delayed for weeks after resolution of acute infection to avoid having

'Department of Otorhinolaryngology, University of Brescia, Italy

${ }^{2}$ Department of Ophthalmology, University of Brescia, Italy

This article was presented at the 20I3 AAO-HNSF Annual Meeting and OTO EXPO; September 29-October 3, 2013; Vancouver, British Columbia, Canada.

\section{Corresponding Author:}

Davide Lombardi, MD, Department of Otorhinolaryngology, University of Brescia, Piazza Spedali Civili I, 25I00 Brescia, Italy.

Email: davinter@libero.it 

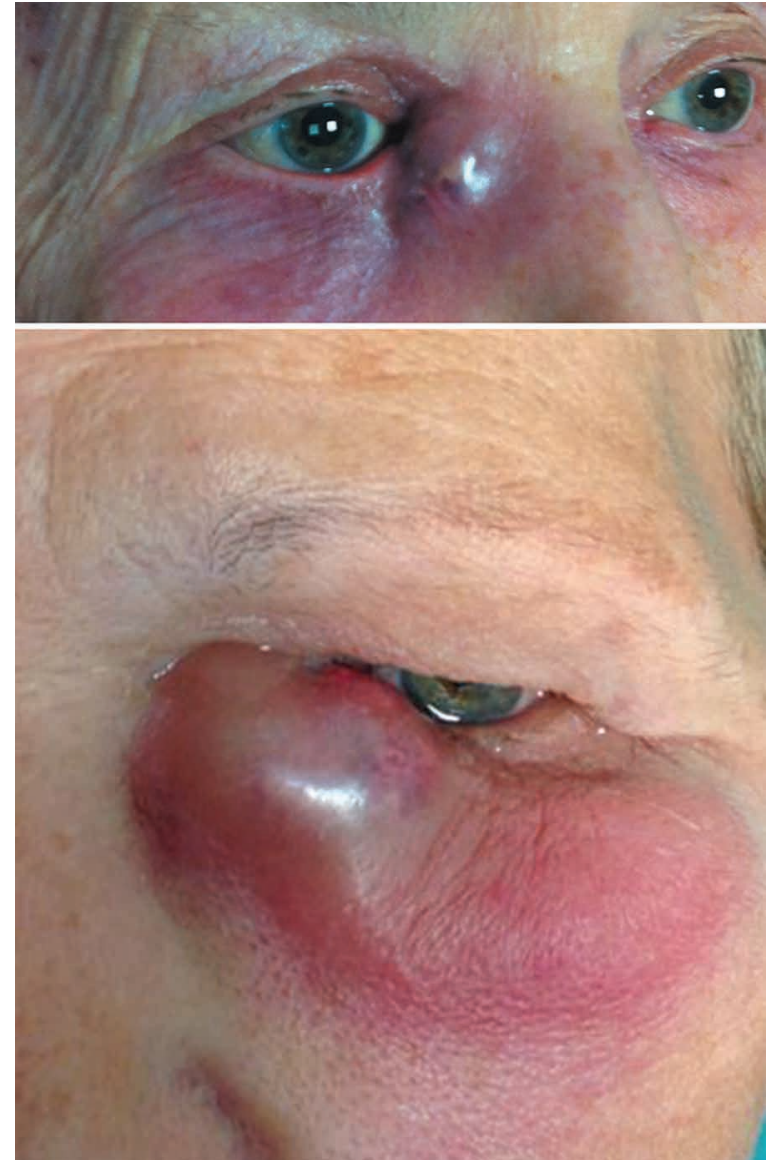

Figure I. Typical clinical appearance of acute dacryocystitis with lacrimal sac empyema (ADLSE) with marked, ill-defined swelling in margins and swelling at the medial cantus. Hyperemic skin covering the enlarged lacrimal sac and eyelid edema may also be present.

to make a skin incision in an infected field $d^{1,3,4}$ and possibly spreading the infection along tissue planes. ${ }^{5}$

In the last 15 years, transnasal endoscopic DCR has changed some common trends in managing lacrimal pathway pathologies. Among these, the possibility of treating ADLSE without the need for a delayed, planned surgery is the most relevant. In the presence of ADLSE, immediate transnasal endoscopic DCR (Endo-DCR) may lead to rapid resolution of infection without a skin incision and may also help decrease the duration of antibiotic therapy with a reasonably limited hospitalization time. ${ }^{2}$

The aim of the present study was to evaluate the efficacy of immediate Endo-DCR in the treatment of ADLSE based on a retrospective review of patients treated in a single academic tertiary center.

\section{Materials and Methods}

This study obtained local institutional review board approval. A database dedicated to patients with obstruction of the lacrimal pathway who were evaluated at the Department of Ophthalmology and surgically treated at the University of Brescia Department of Otorhinolaryngology from August 2005 to December 2013 was retrospectively analyzed. Only patients treated by Endo-DCR for ADLSE with a minimum 6-month follow-up were considered eligible for this study. In the same period, all patients with acute dacryocystitis without clinical signs suggesting lacrimal sac empyema were immediately treated with oral antibiotics and antibiotic-steroid eye drops. Delayed Endo-DCR was planned if the diagnostic workup confirmed a lacrimal pathway obstruction.

Information was collected regarding gender, age, previous surgery, clinical history, comorbidities, possible risk factors for lacrimal pathway obstruction and procedural failure (chemotherapy, radiotherapy [RT] on the sinonasal tract, radioiodine treatment, Wegener's granulomatosis, and other autoimmune conditions), and retreatment in case of recurrence. All patients considered eligible for surgery underwent computed tomography of the sinonasal tract. In contrast to elective Endo-DCR, dacryocystography (DCG) was not performed for ADLSE cases, since the occurrence of lacrimal sac abscesses is considered to be a consequence of a postsaccal stenosis. Systemic broad-spectrum intravenous antibiotic treatment was started at admission and was prolonged until discharge; in the presence of marked edema and absence of specific contraindications, intravenous steroid was also administered (methylprednisolone $20 \mathrm{mg}$ intravenously once daily for 1-2 days). Thereafter, oral antibiotics were administered for 8 days.

The main outcomes to evaluate the success of Endo-DCR were complete resolution of the specific complaints (ie, lacrimal sac infection and epiphora) and DCR patency, the latter being assessed in each patient by transnasal endoscopic examination and lacrimal pathway irrigation; when the patient still complained of epiphora, DCG was also performed. Other outcomes such as hospitalization time, periand postoperative complications, and the need for revision surgery were analyzed to assess the efficacy of the approach.

\section{Surgical Indications and Technique}

Surgery was performed under general anesthesia using both $0^{\circ}$ and $45^{\circ}$ telescopes. When a septal spur or deviation prevented access to the sac area, septoplasty was performed. The mucosa corresponding to the projection of the lacrimal sac was infiltrated with $2 \mathrm{~mL}$ of adrenaline diluted 1:100,000, and a posteriorly pedicled mucoperiosteal flap was harvested and posteriorly displaced. Once the lacrimal bone and the adjacent portion of the maxillary sinus frontal process were exposed, the bone overlying the lacrimal sac was removed. Once the entire medial wall of the lacrimal sac was exposed, a reverse $\mathrm{H}$-shape incision was made on the medial wall of the lacrimal sac with an angled knife (Figure 2). The 2 resulting mucosal flaps were then gently folded anteriorly and posteriorly to cover the bone around the sac itself (Figure 3A). The previously harvested mucoperiosteal flap was anteriorly rotated and then longitudinally incised to obtain 2 smaller flaps that were used to cover the bone superiorly and inferiorly to the sac (Figure 3B and Figure 4). The flaps were positioned with smooth instruments, and a small amount of absorbable haemostatic 


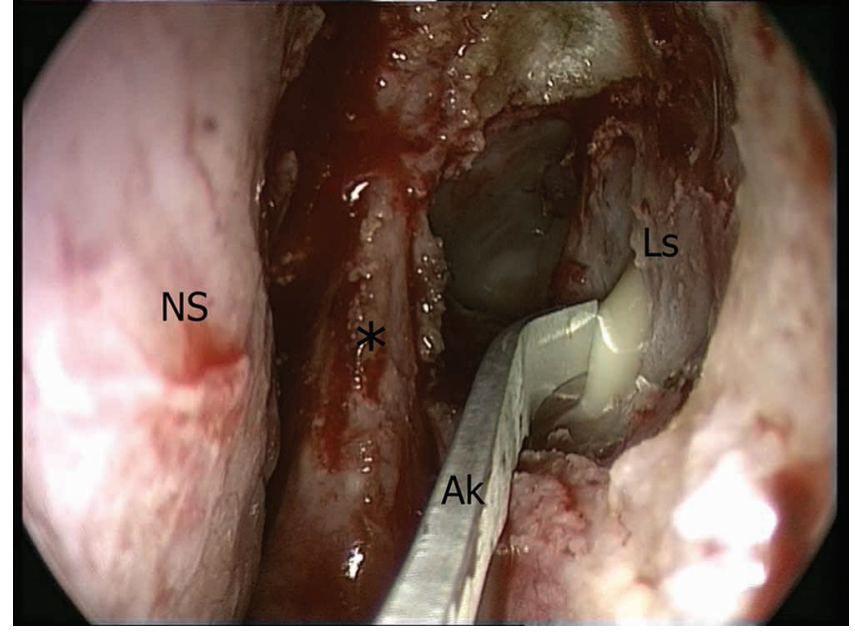

Figure 2. Nasal endoscopy on left side, $0^{\circ}$ endoscope: After its complete exposure, the lacrimal sac medial wall (Ls) is incised by an angled knife (Ak) with evidence of pus drainage. NS, nasal septum. Asterisk indicates posteriorly rotated mucosal flap.
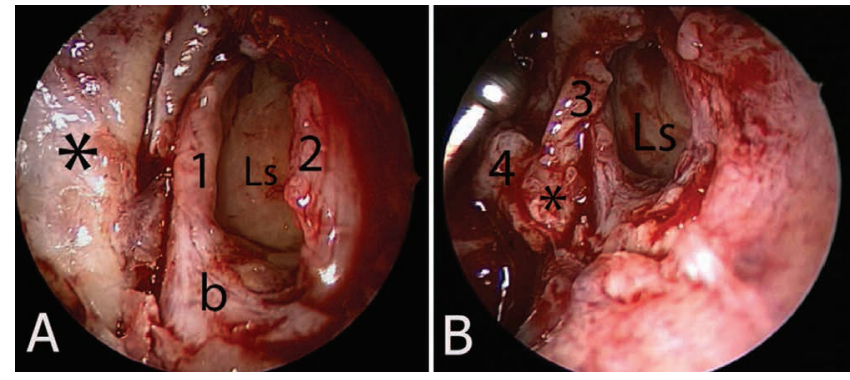

Figure 3. (A) Nasal endoscopy on left side, $45^{\circ}$ endoscope: Once the sac is incised, the mucosa obtained from the lacrimal sac (Ls) medial wall is folded to cover the bone posteriorly (I) and anteriorly (2) to the sac itself. The bone (b) inferior to the sac will be covered with the mucosal flap (asterisk) that will be anteriorly rotated. (B) Nasal endoscopy on left side, $45^{\circ}$ endoscope: The previously harvested mucosal flap (asterisk) is longitudinally incised to obtain 2 smaller flaps that will be used to cover the bone superiorly (3) and inferiorly (4, which will be rotated and positioned) to the lacrimal sac (Ls).

gelatin sponge (Spongostan, Ethicon Inc, Somerville, New Jersey) was used to keep them in place.

A lacrimal stent was positioned in selected cases such as difficult revision surgery, granulomatous diseases, small sac size, and previous RT or radioiodine treatment and in patients with limited compliance or who were unavailable for regular follow-up. In the absence of risk factors for bleeding, nasal packing was not used.

\section{Postoperative Surveillance and Follow-up}

On the first postoperative day, an endoscopic examination of the nasal cavity was performed to remove fibrin and blood clots and to check the patency of the DCR. Lacrimal pathway irrigation was performed in all cases except those with a lacrimal stent. After discharge from the hospital, the

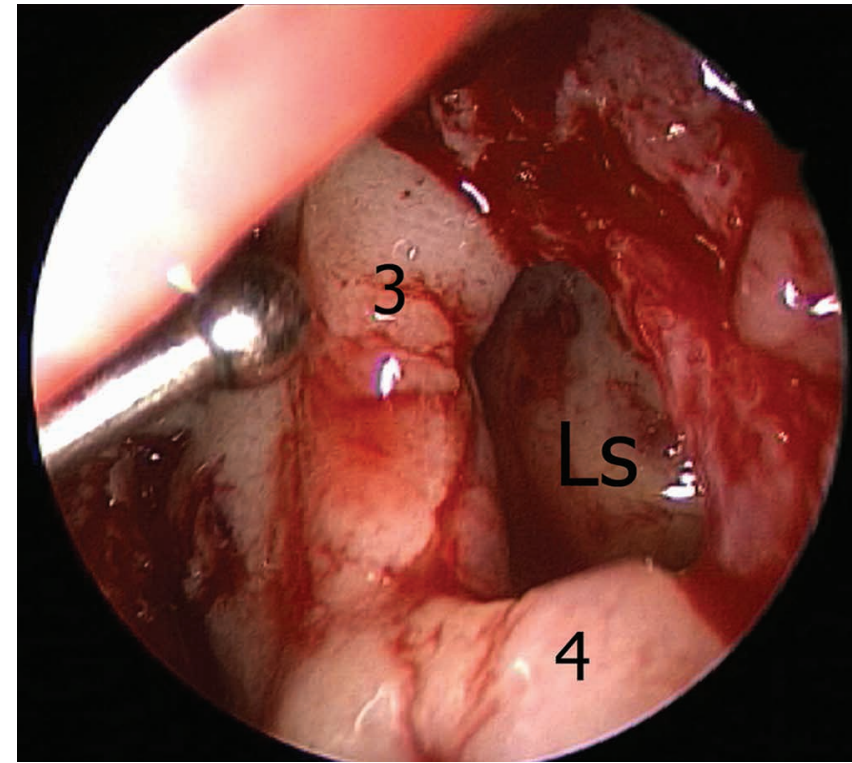

Figure 4. Nasal endoscopy on the left side, $45^{\circ}$ endoscope: Surgical field at the end of the procedure with the 2 last mucosal flaps in place and no residual bone exposed. Ls, lacrimal sac; 3 and 4 , third and fourth flap obtained by longitudinal incision of the previously harvested mucosal flap.

patient was instructed to perform daily nasal irrigations with saline solution and to apply emollient ointment twice daily for 2 weeks. In addition, antibiotic-steroid eye drops were administered twice daily for 2 weeks. Endoscopic debridement and lacrimal irrigations were repeated on the seventh postoperative day. In the presence of satisfactory DCR healing, the patient was scheduled for regular endoscopic evaluations (every 3 months for the first year, every 6 months for the second year). In the presence of abundant scar tissue and/or irregular DCR healing, a stricter follow-up was scheduled. The stent was kept in place for 1 to 3 months depending on associated pathological factors, length of healing process, and sac size.

According to the classification of Zuercher et $\mathrm{al}^{6}{ }^{6}$ complete success was defined as DCR patency upon transnasal endoscopic examination with complete resolution of symptoms. Symptom relief with DCR obstruction was considered a functional success, whereas DCR patency and persistent complaints were defined as an anatomic success. Finally, the association of DCR stenosis with persistent complaints was considered a surgical failure. As previously stated, all cases were evaluated by endoscopy and lacrimal irrigation, and in the presence of epiphora, DCG was planned to assess the site of the obstruction.

\section{Results}

The analysis included 4 (15.4\%) males and 22 (84.6\%) females with a mean age of 66 years (range, 28-98 years). The most frequently reported complaints were painful swelling of the medial canthus and inferior eyelid associated with epiphora. 


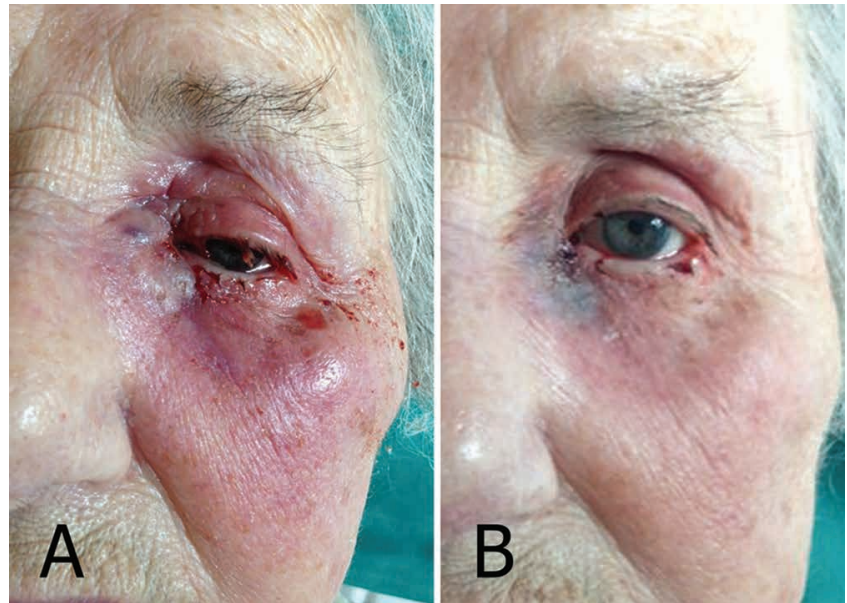

Figure 5. Clinical appearance of a patient with ADLSE the day before $(A)$ and after $(B)$ surgery; the medial canthus swelling and skin hyperemia are considerably reduced.

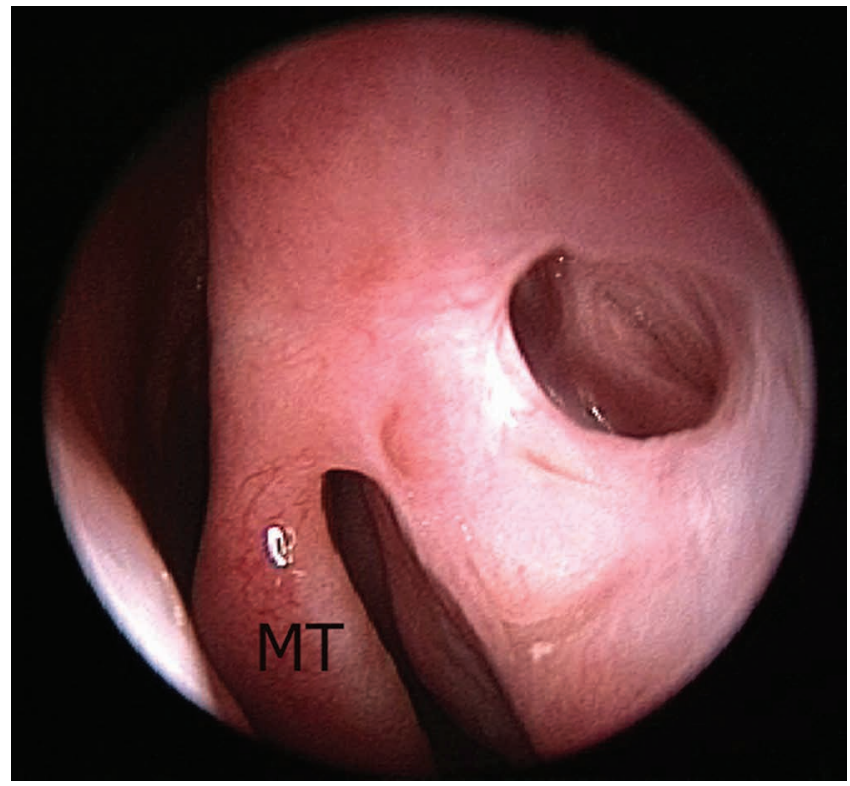

Figure 6. Nasal endoscopy on left side, $45^{\circ}$ endoscope. Endoscopic appearance of a perfectly healed, broad dacryocystorhinostomy without exposure of residual bone. MT, middle turbinate.

The clinical history included up to 20 previous episodes of acute dacryocystitis treated with various combinations of antibiotics and in $5(19.2 \%)$ cases drainage with percutaneous aspiration and/or surgical incision. Only $2(7.7 \%)$ patients had previously submitted to Ex-DCR. Risk factors for lacrimal pathway obstruction were identified in 6 (23.1\%) patients: chemotherapy in 3 patients $(11.5 \%)$ and external beam RT, radioiodine treatment, and psoriatic arthritis in 1 patient each $(3.8 \%)$.

The mean time between referral and surgery was 0.88 days (range, 0-3 days), such that the majority of patients underwent Endo-DCR on the same day as admission. Septoplasty was required in $3(11.5 \%)$ patients. The mean hospitalization time was 2.54 days (range, 1-6 days), while the mean and median follow-up time was 29 and 18 months, respectively (range, 6-102 months).

All patients reported immediate relief from pain and gradual improvement of medial canthus swelling and edema (Figure 5). In 10 (38.5\%) cases, a bicanalicular silicone tube was positioned, either because of the presence of risk factors for procedural failure (5 cases, 19.2\%) or because the patients were considered to be poorly suitable for postoperative medications (5 cases, 19.2\%). The tube was removed after a mean period of 30 days. In term of complications, only one 83 -year-old woman (3.8\% of cases), who had also undergone septoplasty, presented postoperative epistaxis, which was successfully controlled by endoscopic coagulation under general anesthesia. This patient had diffuse bleeding from the nasal septum and lateral nasal wall mucosa that required revision under general anesthesia due to very low patient compliance.

No recurrence of ADLSE was observed in the follow-up period; 3 additional patients were not included in the analysis due to a follow-up period that was shorter than 6 months. Complete functional success was achieved in 25 (96.2\%) patients (Figure 6). The only failure with DCR obstruction and persistent epiphora occurred in a woman who had undergone radioiodine treatment for thyroid gland carcinoma 12 years before Endo-DCR. The first Endo-DCR was performed on the left side without positioning a stent. The patient recovered from pain and no recurrence of ADLSE was described, although she was referred with persistent mild epiphora thereafter. Transnasal endoscopic examination and DCG confirmed that there was an obstruction of the DCR. Two years after the primary treatment, revision of the left DCR was performed and a silicone tube positioned. The tube on the left side was removed 6 months later, but unfortunately the revision DCR also failed. A DCG performed 1 year after revision surgery showed the presence of pre-saccal stenosis.

\section{Discussion}

In recent years, considerable efforts have been made to determine whether Ex-DCR or Endo-DCR is preferred for treating lacrimal pathway obstruction. To date, the common opinion is that the 2 approaches, whenever properly planned and performed, are characterized by comparable functional outcomes. $^{7,8}$

The comparison should therefore entail some adjunctive factors, such as the cosmetic importance of a facial skin incision and scar. Even though a report focusing on this specific issue demonstrated that only a very limited number of patients (4\%) were worried about a scar following ExDCR, ${ }^{9}$ the risk of bad scar formation undeniably exists and is often unpredictable. ${ }^{2}$ Moreover, at least in our experience, many patients, when presented with 2 approaches that have comparable functional outcomes, are more inclined to prefer the one leaving no visible scar. Endo-DCR may also allow concomitant correction of sinonasal anomalies (septal deviation) that could compromise the functional outcome. ${ }^{10}$ 
Finally, additional advantages of a transnasal endoscopic procedure include a magnified surgical field and avoidance of medial canthus ligament sectioning with consequent pump mechanism preservation. ${ }^{11,12}$

Endo-DCR is considered feasible even in the presence of an ADLSE with the possibility of immediately treating abscesses, rapid resolution of infection, limited hospitalization time, and no need for planned lacrimal surgery. ${ }^{2}$

In the past, some experiences supported immediate ExDCR as a reliable option for treating ADLSE. ${ }^{13}$ However, to date the general tendency for those who prefer an external approach is to administer antibiotics and perform surgery only after the infection is resolved in order to avoid making a skin incision in an infected field and spreading inflammation through the dissected planes. ${ }^{14}$

When dealing with ADLSE, the preference for EndoDCR is based on the possibility of immediate treatment offered by a transnasal approach. We agree with Madge et $\mathrm{al}^{2}$ that this procedure is not, in itself, an emergency surgery needed within hours since ADLSE is not a lifethreatening condition. However, our preference for immediate surgery is dictated by 2 main reasons: The risk of skin fistulization, which could result in undesirable scarring in the medial canthus area, is reduced, and immediate intervention can decrease the risk of orbital complications that, despite their rarity, may have catastrophic consequences. Such complications typically include cellulitis and subperiosteal, extra- and intraconal abscesses, the latter possibly leading to optic neuropathy with risk of vision disturbance and even loss. ${ }^{15,16}$

In our experience, treatment of ADLSE with early intravenous broad-spectrum antibiotic administration and immediate Endo-DCR was effective. Endo-DCR allows exposure and opening of the lacrimal sac on the nasal side, thereby reducing dissection in infected and inflamed soft tissues. The risk of orbital complications and the duration of treatment and hospitalization are therefore reduced. Even the risk of bleeding following surgery in an infected field is very low. We experienced only 1 postoperative epistaxis; this complication, as already stated, was a diffuse venous rather than an arterial bleeding that, during endoscopic DCR, is generally related to a lesion of the angular artery or a branch of the anterior ethmoidal artery.

Regarding economic benefits, patients treated with early Endo-DCR were shown to have significantly shorter hospitalization time than those treated according to a "conventional" schedule, with important implications in terms of cost and patient morbidity. ${ }^{2}$ However, the total health costs of our approach, including hospitalization time and postoperative surveillance, were not assessed, and the costeffectiveness ratio of our protocol should be better defined, possibly in a prospective way.

When one is analyzing the functional results of EndoDCR in ADLSE, there are 3 main outcomes to be evaluated: absence of relapsing lacrimal sac infection, resolution of epiphora, and DCR patency. As highlighted by Madge et $\mathrm{al}^{2}{ }^{2}$ in the 5 publications that focus on this topic, the results of Endo-DCR in the treatment of ADLSE are not uniform. ${ }^{2,4,10,17,18}$ Notably, in these studies complete resolution of lacrimal sac infection was achieved in all cases, although epiphora and lacrimal pathway obstruction persisted at rates ranging from $5.6 \%$ to $33 \%{ }^{2,4,10,17,18}$ Lee and Woog ${ }^{17}$ analyzed 24 patients treated endoscopically with a hybrid technique (powered drill and then YAG laser) and found that ostium patency and absence of epiphora were obtained in $83 \%$ of patients. Causes of failure were identified as granuloma formation around the ostium in 1 case and membranous obstruction of the ostium in the remaining 3 cases. Morgan et $\mathrm{al}^{10}$ performed laser-assisted endoscopic DCR in 9 patients with acute dacryocystitis and obtained functional and anatomical success in only $67 \%$ of cases, with the remaining 3 cases having persistent complaints and showing a stenotic ostium with fibrous tissue. Duggal et al ${ }^{4}$ treated 11 patients with acute dacryocystitis with abscess formation by endoscopic DCR under local anesthesia using cold instruments; subjective improvement of symptoms and lacrimal pathway patency was achieved in $72.7 \%$ and $81.8 \%$ of patients, respectively. Wu et al, ${ }^{18}$ in a prospective randomized study, analyzed a cohort of 72 patients with acute dacryocystitis, of whom 40 were treated endoscopically and 32 were treated by an external approach, with the second group of patients treated 1 to 2 weeks after resolution of acute inflammation. Ostium patency was achieved in $90 \%$ of patients treated endoscopically and in $65.7 \%$ of cases in the external group. Notably, the duration of acute inflammation was significantly shorter with an endoscopic approach. Causes of failure following an endoscopic procedure were intranasal ostium closure and common canaliculus obstruction in 3 and 1 case, respectively.

Madge et $\mathrm{al}^{2}{ }^{2}$ in their retrospective, multicenter study, reported excellent results with an endoscopic approach, as $94.4 \%$ of patients were free of epiphora with a patent lacrimal pathway. The only failure was observed in a patient for whom, due to intraoperative bleeding, the status of the mucosal flaps was suboptimal.

Our results compare favorably with the data in the literature, with $96.2 \%$ of patients in this study having no residual complaints. Some speculations may be offered to explain this favorable outcome. Considering surgical technique, wide exposure of the lacrimal sac medial wall and the use of mucosal flaps to cover the drilled bone may have played a role in minimizing the healing process. The incomplete exposure of the sac leads to harvest of a small DCR, with the consequent risk of occlusion. From this perspective, it is essential to completely remove the bone over the sac with a drill or cutting instruments. Our surgical technique, which has been slightly modified over the years, now entails the use of a diamond-bur $(4 \mathrm{~mm})$ with intermittent saline irrigation to minimize heat damage. Similarly, to reduce heat damage to soft tissues, we do not use a laser to harvest the mucosal flap or incise the lacrimal sac wall. Satisfactory exposure also requires preventive correction of possible anatomic variants, such as septal deviation, before approaching the sac, thus favoring not only the surgical procedure but 
also postoperative medications. Moreover, the presence of an agger nasi cell medial to the lacrimal sac as well as the modality of insertion of the uncinate process on the lateral nasal wall must be preoperatively assessed. ${ }^{19}$ According to the technique described by Tsirbas and Wormald, ${ }^{11}$ we also elevate a posteriorly based mucoperiosteal flap before removing the bone covering the medial wall of the lacrimal sac.

Thereafter, minimizing the area of exposed bone around the sac that remains uncovered at the end of surgery is essential, since this is precisely the site where a granuloma is more likely to develop. We generally cover the bone by mucosal rotational flaps according to a technique that, with some minor differences, has already been described and is intended to favor primary intention healing. ${ }^{11,12,20,21}$ When allowed by anatomic conditions, we harvest 4 flaps, with 2 obtained by extroversion of the lacrimal sac mucosa and 2 by a posteriorly based mucoperiosteal flap.

When one is treating ADLSE, the large size of the lacrimal sac may be helpful in obtaining sufficient mucosal tissue to cover the bone and reducing the risk of ostium shrinkage and obstruction. In contrast, when limited lacrimal sac dimensions are present, the 2 flaps obtained by the mucoperiosteal flap are harvested and positioned, and with such a condition, a lacrimal bicanalicular stent may also be helpful. Interestingly, in the literature there is no general consensus about the use of mucosal flaps, and recent publications have reported excellent functional results without harvesting them. ${ }^{22}$ Notably, the only failure we described in this study occurred in a woman in whom a well-known risk factor for lacrimal pathway restenosis was identified. This patient reported resolution of acute dacryocystitis, whereas epiphora persisted despite revision surgery and stent positioning. DCG performed after stent removal revealed the presence of a pre-saccal stenosis that might have resulted from improper lacrimal pathway management during irrigations or surgery or pre-saccal pathway involvement by the inflammatory process that gave rise to acute dacryocystitis. It is possible that some of the patients reported in the literature with patent DCR and persistent epiphora, but not recurrent lacrimal sac infection, may also have had an occult presaccal stenosis.

\section{Conclusion}

The present study demonstrates that Endo-DCR enables a rapid resolution of ADLSE with a very high success rate. Immediate surgery reduces the need for prolonged therapy and may prevent skin fistulization and/or orbital complications. DCR shrinkage and lacrimal obstruction are unlikely since the procedure is performed on an enlarged sac. While our study lacks a control group treated with a more conservative approach, given the excellent outcome of our treatment protocol, we would consider it unethical to offer patients an alternative treatment that might include an external incision of the sac to drain the empyema and/or an external DCR.

\section{Author Contributions}

Davide Lombardi, conception and design, interpretation of data, drafting of the manuscript, final approval; Davide Mattavelli, acquisition and interpretation of data, drafting of the manuscript, final approval; Remo Accorona, acquisition of data, drafting of the manuscript, final approval; Raffaele Turano, acquisition of data, drafting of the manuscript, final approval; Francesco Semeraro, conception and design, revision of the manuscript, final approval; Anna Bozzola, acquisition of data, drafting of the manuscript, final approval; Piero Nicolai, conception and design, revision of the manuscript, final approval.

\section{Disclosures}

Competing interests: None.

Sponsorships: None.

Funding source: None.

\section{References}

1. Cahill KV, Burns JA. Management of acute dacryocystitis in adults. Ophthal Plast Reconstr Surg. 1993;9:38-41.

2. Madge SN, Chan W, Malhotra R, et al. Endoscopic dacryocystorhinostomy in acute dacryocystitis: a multicentric case series. Orbit. 2011;30:1-6.

3. Massaro BM, Gonnering RS, Harris GJ. Endonasal laser dacryocystorhinostomy: a new approach to nasolacrimal duct obstruction. Arch Ophthalmol. 1990;108:1172-1186.

4. Duggal P, Mahindroo NK, Chauhan A. Primary endoscopic dacryocystorhinostomy as treatment for acute dacryocystitis with abscess formation. Am J Otolaryngol. 2008;29:177-179.

5. Coden DJ, Hornblass A, Haas BD. Clinical bacteriology of dacryocystitis in adults. Ophthal Plast Reconstr Surg. 1993;9: 125-131.

6. Zuercher B, Tritten JJ, Friedrich JP, Monnier P. Analysis of functional and anatomic success following endonasal dacryocystorhinostomy. Ann Otol Rhinol Laryngol. 2011;120:231-238.

7. Eichhorn K, Harrison AR. External vs. endonasal dacryocystorhinostomy: six of one, a half dozen of the other? Curr Opin Ophthalmol. 2010;21:396-403.

8. Karim R, Ghabrial R, Lynch TF, Tang B. A comparison of external and endoscopic endonasal dacryocystorhinostomy for acquired nasolacrimal duct obstruction. Clin Ophthalmol. 2011;5:979-989.

9. Sharma V, Martin PA, Benger R, et al. Evaluation of the cosmetic significance of external dacryocystorhinostomy scars. Am J Ophthalmol. 2005;140:359-362.

10. Morgan S, Austin M, Whittet $H$. The treatment of acute dacryocystitis using laser assisted endonasal dacryocystorhinostomy. Br J Ophthalmol. 2004;88:139-141.

11. Tsirbas A, Wormald PJ. Mechanical endonasal DCR with mucosal flap. Am J Ophthalmol. 2003;135:79-86.

12. Trimarchi M, Giordano Resti A, Bellini C, Forti M, Bussi M. Anastomosis of nasal mucosal and lacrimal sac flaps in endoscopic dacryocystorhinostomy. Eur Arch Otorhinolaryngol. 2009;266:1747-1752.

13. Iliff CE. A simplified dacryocystorhinostomy: 1954 to 1970. Trans Am Acad Ophthalmol Otolaryngol. 1971;75:821-828. 
14. Ari S, Cingu K, Şahin A, Ozkok A, Caca I. The outcomes of surgical treatment in fistulous dacryocystitis. Eur Rev Med Pharmacol Sci. 2013;17:243-246.

15. Kikkawa DO, Heinz GW, Martin RT, Nunery WN, Eiseman AS. Orbital cellulitis and abscess secondary to dacryocystitis. Arch Ophthalmol. 2002;120:1096-1099.

16. Maheshwari R, Maheshwari S, Tarjani T. Acute dacryocystitis causing orbital cellulitis and abscess. Orbit. 2009;28:196-199.

17. Lee T, Woog J. Endonasal dacryocystorhinostomy in the primary treatment of acute dacryocystitis with abscess formation. Ophthal Plast Reconstr Surg. 2001;17:180-183.

18. Wu W, Wentao Y, MacCallum JK, et al. Primary treatment of acute dacryocystitis by endoscopic dacryocystorhinostomy with silicon intubation guided by a soft probe. Ophthalmology. 2009;116:116-122.

19. Soyka MBN, Treumann T, Schlegel CT. The Agger Nasi cell and uncinate process, the keys to proper access to the nasolacrimal drainage system. Rhinology. 2010;48:364-367.

20. Wormald PJ. Powered endoscopic dacryocystorhinostomy. Laryngoscope. 2002;112:69-72.

21. Codere F, Denton P, Corona J. Endonasal dacryocystorhinostomy: a modified technique with preservation of the nasal and lacrimal mucosa. Ophthal Plast Reconstr Surg. 2010;26:161-164. 22. Ramakrishnan VR, Hink EM, Durairaj VD, Kingdom TT. Outcomes after endoscopic dacryocystorhinostomy without mucosal flap preservation. Am J Rhinol. 2007;21:753-757. 